\title{
Electron-beam-induced optical memory effects in GaN
}

\author{
Y. C. Chang, A. L. Cai, M. A. L. Johnson, J. F. Muth, and R. M. Kolbas ${ }^{a)}$ \\ Department of Electrical and Computer Engineering, North Carolina State University, Raleigh, \\ North Carolina 27695-7911 \\ Z. J. Reitmeier, S. Einfeldt, and R. F. Davis \\ Department of Materials Science and Engineering, North Carolina State University, Raleigh, \\ North Carolina 27695-7907
}

(Received 6 November 2001; accepted for publication 13 February 2002)

\begin{abstract}
Metastable effects in unintentionally doped $\mathrm{GaN}$ films grown on $\mathrm{SiC}$ substrates have been investigated using cathodoluminescence (CL). Memory effect patterns produced optically are observed in CL images. An electron beam can also produce memory effect patterns and the resulting changes in the luminescence spectra are quite similar for either optical or electron-beam-induced patterns. CL spectra reveal that the yellow luminescence at $2.2 \mathrm{eV}$ increases significantly with little change in the band-edge emission in both cases. Samples that do not exhibit optically induced memory effects are also investigated and do not exhibit electron-beam-induced patterns, either. Monochromatic CL images at 540 and $365 \mathrm{~nm}$ confirm the similarity of optically and electron-beam-induced memory effects based on changes in luminescence spectra. (c) 2002 American Institute of Physics. [DOI: 10.1063/1.1469222]
\end{abstract}

$\mathrm{GaN}$ and its ternary alloys are of great interest for applications in high-temperature/high-power/high-frequency electronics, ${ }^{1,2}$ visible-blind photodetectors, ${ }^{3}$ and UV blue emitters. ${ }^{4}$ Consequently, intense research has been focused on how to improve the material quality as well as the device performance of III-V nitrides.

The performance of an optoelectronic device can be influenced by parasitic defect-related emissions such as the well-known yellow luminescence (YL) observed in undoped GaN. Photoinduced metastable effects could also alter the device performance via changes in optical emission, absorption, and electron transport. ${ }^{5}$ Optical metastability in bulk GaN single crystal has been observed. ${ }^{6}$ Reconfigurable optical properties have been observed in an $\mathrm{InGaN} / \mathrm{GaN}$ heterostructure ${ }^{7}$ and $\mathrm{InGaN} / \mathrm{GaN}$ quantum wells ${ }^{8}$ grown on sapphire substrates. Similar effects were also observed in undoped $\mathrm{GaN}$ grown on $\mathrm{SiC}$ substrates. ${ }^{9}$ The distinguishing characteristic of this effect is that the YL peak intensity at 2.2 $\mathrm{eV}$ increases dramatically in the regions where the sample was illuminated by intense UV laser light. A yellow pattern is observed under low intensity UV light illumination for several minutes/hours depending on the sample temperature. ${ }^{9}$

Photography and photoluminescence (PL) have been the principle techniques used to record and measure this metastable effect. In this letter, we report the writing and reading of memory effect patterns on $\mathrm{GaN}$ by an electron beam and cathodoluminescence (CL). The resulting changes in the luminescence spectra are quite similar for either optical or electron beam induced patterns. In both cases, the YL at 2.2 $\mathrm{eV}$ increases significantly with little change in the band-edge emission. For the samples used in this study, the memory effect self-erases in several hours at room temperature or can be erased in a few seconds at $110^{\circ} \mathrm{C}$.

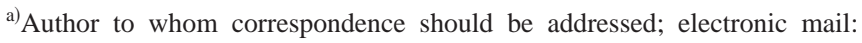
kolbas@eos.ncsu.edu
}

The GaN layers used in this study were deposited on $6 \mathrm{H}-\mathrm{SiC}(0001)$ substrates in a vertical, resistively heated, cold walled quartz reactor equipped with a rotating $\mathrm{SiC}$ coated graphite stage operating at $300 \mathrm{rpm}$. Hydrogen was employed as the diluent and carrier gas. Prior to GaN deposition, a $0.1 \mu \mathrm{m}$ AlN buffer layer was grown at a chamber pressure of 45 Torr and a temperature of $1150{ }^{\circ} \mathrm{C}$ using trimethylaluminum and $\mathrm{NH}_{3}$. The temperature was then lowered to $1040^{\circ} \mathrm{C}$ for $\mathrm{GaN}$ growth. The samples that exhibit optical memory effects were grown to a thickness of $1 \mu \mathrm{m}$ using trimethylgallium and $\mathrm{NH}_{3}$. The sample that did not exhibit optical memory effects consisted of an AlN buffer followed by a $0.05 \mu \mathrm{m}$ undoped GaN layer and finally a 1 $\mu \mathrm{m}$ Si-doped GaN layer. The silicon concentration as measured by secondary ion microscopy is $3 \times 10^{18}\left(\mathrm{~cm}^{-3}\right)$.

A scanning electron microscope (SEM) equipped with a digital scan generator and a CL accessory was used to characterize the samples. CL from 180 to $900 \mathrm{~nm}$ can be obtained based on either the total integrated CL intensity (panchromatic) or monochromatic CL images of the samples. Detailed information about this SEM/CL system can be found in Ref. 10.

The frequency tripled output of a pulsed Ti: Sapphire laser $(280 \mathrm{~nm}$ and $250 \mathrm{fs}$ at $76 \mathrm{MHz}$ ) was used as the UV excitation source. Writing images (lines) on the samples was accomplished by focusing the ultraviolet source to a spot size of approximately $20 \mu \mathrm{m}$ which produced an average power density of $30 \times 10^{3} \mathrm{~W} / \mathrm{cm}^{2}$. In the read condition, the sample was illuminated at $P_{\mathrm{AVE}}=0.4 \mathrm{~W} / \mathrm{cm}^{2}$ with the same UV laser source. Schematic views of the write and read conditions are shown in Fig. 1(b). Additional information regarding the read and write conditions can be found in Refs. 6 and 7.

The appearance of the sample through an optical microscope in the read condition is shown in Fig. 1(a). The pattern is somewhat out of focus because of the high magnification and the off axis angle of the microscope during the experi- 

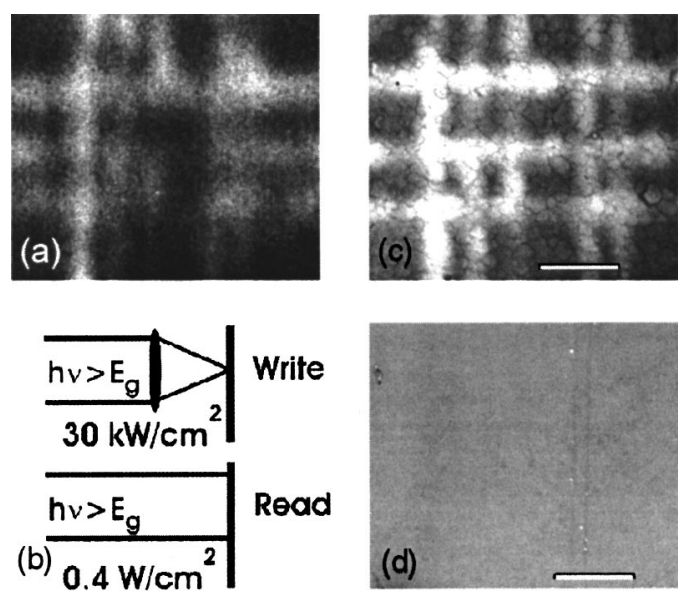

FIG. 1. Room temperature images $(320 \mu \mathrm{m} \times 380 \mu \mathrm{m} ; 100 \mu \mathrm{m}$ reference marker) using different measurement techniques to view the memory effect pattern that is produced by intense laser light. Three horizontal and five vertical lines were optically written using the conditions shown in (b). The effect is clearly observed under optical "read" conditions (a) and panchromatic CL (c), but not by secondary-electron imaging (d) or optical microscopy (not shown).

ment. Regardless, five vertical and three horizontal parallel lines can be observed. The sample was then transferred into the SEM/CL system. Panchromatic CL images and secondary-electron images were taken with a probe current of $6 \times 10^{-10} \mathrm{~A}$ and acceleration voltage of $5 \mathrm{kV}$ in the same area where the photo was taken. The panchromatic CL image, shown in Fig. 1(c), clearly shows the same pattern that is observed under the optical microscope in the read condition. A secondary-electron image, shown in Fig. 1(d), does not reveal the pattern. These images indicate that intense laser light illumination on the samples temporarily changes the material property and effects how excess carriers, generated either by laser light or electron beam, recombine. The higher brightness from the pattern indicates that more luminescence signal is collected in the patterned area.

The PL spectra on (bright) and off (dark) the written patterns are shown in Figs. 2(a) and 2(b), respectively. Note that the peak intensity at $550 \mathrm{~nm}$ increases while the intensity of the band-to-band transition is almost the same. The CL spectra on the bright and dark areas of Fig. 1(c) are shown as curves 2(c) and 2(d) in Fig. 2-2. Again, the peak intensity centered at $550 \mathrm{~nm}$ is higher and the band-edge emission is nearly equal as in the PL spectra. The CL spectra are consistent with the CL images shown in Fig. 1(c).

Samples with and without optical memory effect characteristics were selected to determine if an electron beam could be used to write memory effect patterns. To produce a highdensity electron beam, the magnification of the image is changed to $1600 \times$ and the probe current is increased to 6 $\times 10^{-9}$ A. Each sample was exposed to this high-intensity electron beam for about 20 minutes. The magnification of the images was subsequently changed to $500 \times$ and the probe current was turn down to $10^{-10}$ A. Panchromatic CL images taken under this condition (write current density $\approx 600$ $\times$ read current density) are shown in Figs. 3(a) and 3(b). Secondary-electron images, Figs. 3(c) and 3(d), on the same locations were taken immediately after obtaining the CL images. Figure 3(a) is the panchromatic CL image of a sample that exhibited optical memory effects. Note that the electronDownloaded 16 Apr 2008 to 152.1.24.180. Redistribution subject to
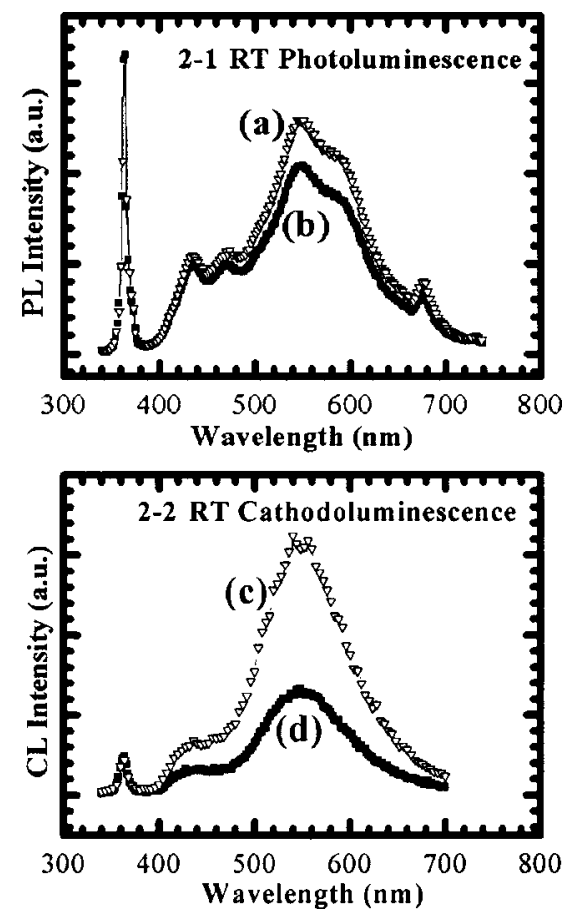

FIG. 2. Comparison of room temperature PL (upper graph) and CL (lower graph) spectra before and after the memory effect pattern is optically produced in the "Write" condition. (a) PL-after, (b) PL-before, (c) CLafter, and (d) CL-before.

beam irradiated rectangular area is brighter than the rest of the sample. Hence, it is possible to generate electron-beam induced memory effects like those induced by optical means. Using the same experimental conditions, a Panchromatic CL image of a sample that did not exhibit optical memory effects is shown in Fig. 3(b). The exposed area is diminished in intensity leaving a faint image. This image is caused by the buildup of hydrocarbons on the surface after a long exposure to the electron beam. The hydrocarbon buildup, while thin, attenuates the emission from the sample. This effect is quite common and can also be observed in the secondary-electron images on both samples [Fig. 3(c) and 3(d)].

Monochromatic CL images of the pattern produced by
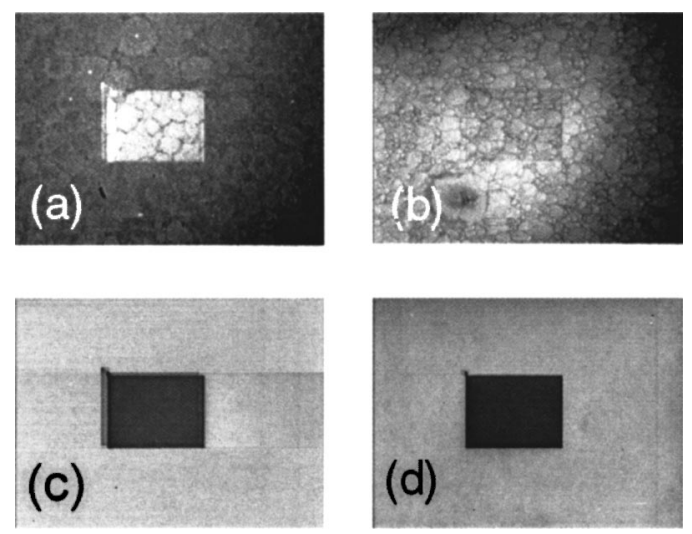

FIG. 3. Panchromatic CL images and secondary-electron images, on samples that did (a) or did not (b) exhibit optically induced memory effects, after high-intensity electron beam irradiation. Electron-beam irradiation produces enhanced yellow emission on a sample exhibiting memory effects (a) while producing a dark image on a sample that did not exhibit optical memory effects (b). Secondary-electron images of both samples reveal a dark area (buildup due to carbon) that was not observed in Fig. 1(a). The

size of the rectangle is approximately $60 \mu \mathrm{m} \times 80 \mu \mathrm{m}$. 

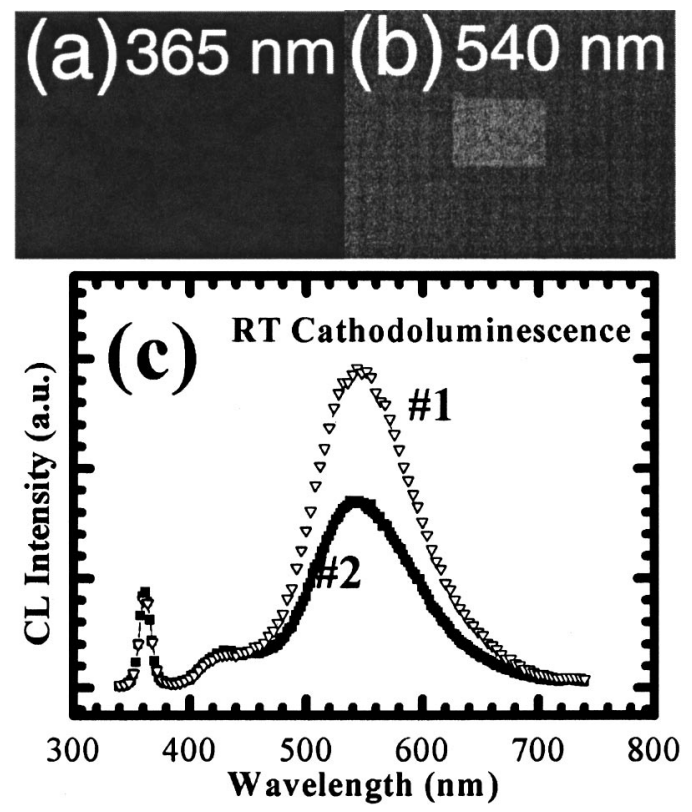

FIG. 4. Monochromatic CL images from a sample exhibiting memory effects after high-intensity electron-beam irradiation. The high contrast in the yellow but not at the band edge is consistent with the CL spectra shown in (c).

the electron beam are shown in Fig. 4(a) for $\lambda=365 \mathrm{~nm}$ and Fig. 4(b) for $\lambda=540 \mathrm{~nm}$. Note that there is an observable contrast in the yellow $(540 \mathrm{~nm})$ but not at the band edge (365 $\mathrm{nm})$. This is consistent with the CL spectra shown in Fig. 4(c). The contrast in CL spectra produced by electron-beam irradiation is very similar to changes in CL spectra induced by optical irradiation. We believe that the same mechanism is responsible in both cases.
In conclusion, optically induced memory effect patterns can be read using either PL or CL. In both cases, emission at $540 \mathrm{~nm}$ is enhanced while band-edge emission remains relatively unchanged. A high-intensity electron beam can also be used to produce (write) memory effect patterns on the same sample. The monochromatic CL images at 365 and $540 \mathrm{~nm}$ reveal that the pattern is caused by increased emission at 540 $\mathrm{nm}$, which corresponds to the classic YL observed from $\mathrm{GaN}$. The CL spectra on the pattern produced by an electron beam is similar to those produced by UV laser light. Memory effect patterns can be written using either high-intensity laser light or a high-intensity electron beam. We believe that charge transfer on and off of defects is responsible for both optically and electron-beam induced memory effects.

${ }^{1}$ H. Morkoc, S. Strite, G. B. Gao, M. E. Lin, B. Sverdlov, and M. Burns, J. Appl. Phys. 76, 1363 (1994).

${ }^{2}$ Y. F. Wu, B. P. Keller, S. Keller, D. Kapolnek, P. Kozodoy, S. P. DenBaars, and U. K. Mishra, Appl. Phys. Lett. 69, 1438 (1996).

${ }^{3}$ A. Osinsky, S. Gangopadhyay, J. W. Yang, R. Gaska, D. Kuksenkov, H. Temkin, I. K. Shmagin, Y. C. Chang, J. F. Muth, and R. M. Kolbas, Appl. Phys. Lett. 72, 551 (1998).

${ }^{4}$ S. Nakamura, T. Mukai, and M. Senoh, Appl. Phys. Lett. 64, 1687 (1994).

${ }^{5}$ G. Beadie, W. S. Rabinovich, A. E. Wickenden, D. D. Koleske, S. C. Binari, and J. A. Freitas, Jr., Appl. Phys. Lett. 71, 1092 (1997).

${ }^{6}$ I. K. Shmagin, J. F. Muth, J. H. Lee, R. M. Kolbas, C. M. Balkas, Z. Sitar, and R. F. Davis, Appl. Phys. Lett. 71, 455 (1997).

${ }^{7}$ I. K. Shmagin, J. F. Muth, R. M. Kolbas, R. D. Dupuis, P. A. Grudowski, C. J. Eiting, J. Park, B. S. Shelton, and D. J. H. Lambert, Appl. Phys. Lett. 71, $1382(1997)$

${ }^{8}$ I. K. Shmagin, J. F. Muth, R. M. Kolbas, M. P. Mack, A. C. Abare, S. Keller, L. A. Colden, U. K. Mishra, and S. P. DenBaars, Appl. Phys. Lett. 71, 1455 (1997)

${ }^{9}$ Y. C. Chang, A. E. Oberhofer, J. F. Muth, R. M. Kolbas, and R. F. Davis, Appl. Phys. Lett. 79, 281 (2001).

${ }^{10}$ Z. Yu, M. A. L. Johnson, T. Mcnulty, J. D. Brown, J. W. Cook, Jr., and J. F. Schetzina, MRS Internet J. Nitride Semicond. Res. 3, 6 (1998). 